\title{
APPROACHES BASED ON ARTIFICIAL INTELLIGENCE FOR WATER SUPPLY SYSTEMS
}

\author{
Gabriela Andrei ${ }^{1}$ \\ ${ }^{1}$ Constanta Maritime University, Faculty of Naval Electro-Mechanics, 104 Mircea cel Batran Street, 900663, Constanta, \\ Romania, e-mail address:gabriela.andrei@cmu-edu.eu
}

\begin{abstract}
In the current context of the existence of life, of the development of human activities, water has a double importance:

- environmental factor, generator of ecological systems

- "raw material" for certain uses (drinking water, industrial water, fish farming, leisure, etc.)

The current problems in the field of water supply are due to:

- exponential increase of water demand;

- the limited water resources and their uneven distribution, which requires large and expensive works of development and accumulation of water;

- deterioration of the quality of water sources, as a result of human activity and the emergence of industries that discharge waste, containing very stable impurities, difficult to remove from water, in the processes of water purification or treatment;

- increase in standards on quality conditions that must be met by water delivered to the population [1].

For the extensive and intensive development of water supplies, a concrete solution, already existing in the area of Artificial Intelligence is given by heuristic methods and Evolutionary Calculus.

This article provides an overview of the role of the most important metaheuristics, based on evolutionary concepts - Evolutionary computation and behavioral patterns inspired by biology - Swarm calculus) in the case of of water supply systems and their subsystems, with exemplification in the case of a model network (Scheme of the network distribution in Hanoi), taking into account water quality (its treatment with chlorine).
\end{abstract}

Key words: The water supply, artificial intelligence, swarm, swarm of ants.

\section{INTRODUCTION}

Swarm Intelligence is a new computational paradigm based on the study of the behaviors of socially organized groups in the animal world (ant colonies, groups of birds, lots of fish, bee colonies, bacterial colonies).

Collective intelligence is based on the exchange of information between members. The interaction between the neighbors, as well as the interactions with the environment, allows them to build complex structures and achieve their goal (the optimal road to certain locations, avoiding predators, etc.). The success of this type of behavior inspired the development of the two metaheuristics: Particle Swarm Optimization (PSO) and Ant Colony Optimization (ACO).

AMF is part of the category of evolutionary stochastic optimization algorithms and is inspired by the search behavior of an ant colony, to detect the food source and bring it to the mound on the shortest path.
This behavior is based on traces of pheromones secreted and deposited by ants in their movements, traces that serve as an indirect form of communication. Routes with higher pheromone intensity are chosen primarily by members of the colony and thus the pheromone concentration on those routes is continuously amplified. In a very general way, the MFA operates with a group of artificial ants, each of them corresponding to a complete solution of the problem to be solved and having a certain quality, in relation to the intended optimization objective. Based on these individual qualities, the intensities of the pheromone are initialized for the possible option variants, ending a global iteration (a search stage or a time step - admitted here discreetly as opposed to the process in nature). In the next global iteration, a new group of ants is generated stochastically (possible solutions), but more favoring the options with increased pheromone intensity. After evaluating them in relation to the objective of the problem and readjusting the intensity of the pheromone for all options, it is 
Journal of Marine Technology and Environment expected that some of them will become stronger and more attractive to be selected in future iterations. The process ceases after an imposed number of global iterations, and the most successful ant (solution) in the last iteration has the chance to place itself very close to the optimal global solution of the problem [4].

An example of implementing this strategy was developed in 2008 by Liana Ioana VUȚĂ and Radu POPA, in search of a model for optimal sizing of hydraulic networks, which includes both the annual costs of investing in pipelines and the costs associated with water chlorination. From a hydraulic point of view, the sizing must comply with the minimum pressure restrictions on the calculation flow regime, estimated as the highest for the respective network.

\section{IMPLEMENTATION OF THE ALGORITHM}

In terms of water quality, a frequency chart imposed during the year is allowed, having different classes of flows to consumers (below the calculation flows) and for each class, is determined the concentration of chlorine required at network entry, the amount of chlorine associated and its cost so that the residual chlorine in the network exceeds a specified minimum concentration. As an optimization method, the extended ant algorithm (AMF), described in detail for the hydraulic part in Popa and Vuţă, 2007 [3], was adopted for this extended problem.

For the formulation of the mathematical model, it is taken into account that, as a rule, when dimensioning the water networks, the positions of the consumption nodes and the required calculation flows, the route of the connection pipes and their lengths, as well as the position of the supply tank and the hydraulic load at the tank are known. The problem is as, from a discrete number of standardized values, choosing the appropriate diameter for each pipe so that at any node in the network to ensure at least a minimum value of the working pressure at the calculation mode, but minimizing the cost of making pipes. In any optimization method, this objective is achieved by selecting the lowest standard diameters (having the lowest unit costs) that lead to compliance with the minimum pressure restrictions imposed.

However, the small diameters imply high water velocities on the pipes, reduced retention times in the system, limited loss of chlorine during transport in the network and therefore the need for a lower chlorine concentration when leaving the tank to ensure a minimum level of residual chlorine required at consumers. This happens when operating in calculation flow mode, and at this mode the low cost of pipes that respond to hydraulic pressure restrictions is associated with the lower costs required for chlorination at the
Year 2021, Vol.I

supply tank. It is known that the distribution network, once built, operates only a limited fraction of the time under the conditions of calculation flows. The lower flows of network determine the lower velocities of water on the pipes and the longer transit times in the system. As a result, the loss of chlorine in the network is amplified, and in order to maintain the minimum permitted level of residual chlorine in consumers, an increased concentration (and therefore higher treatment costs) is required when leaving the tank. In order to take into account both aspects of costs, it is proposed that the optimal sizing analysis be done on an annual basis. In this sense, a specified lifetime, $\mathrm{T}$ years, is allowed for the considered network, and the annual cost corresponding to a possible solution, $\phi$, of pipe diameters, Vh $(\varphi)$, will be taken simply, considering two cases, if the solution $\varphi$ complies with the minimum load restriction imposed, respectively, if the restriction is violated.

$$
{ }_{h}(\varphi)=\left\{\begin{array}{c}
\frac{1}{T} \sum_{i=1}^{I} L_{i} \cdot c_{s i j} \\
\frac{1}{T} \sum_{i=1}^{I} L_{i} \cdot c_{s i j}+p_{h} \cdot \Delta H_{\max }
\end{array}\right.
$$

I este the number of pipes in the network, $\mathrm{Li}$ is the length of the pipe $\mathrm{i}$, cij is the unit cost (per linear meter) of the pipe of standard diameter dij chosen for pipe i, $\Delta$ Hmax represents the maximum pressure deficit in the network compared to the minimum imposed pressure, and ph is a cost penalty factor for violating the minimum service pressure restriction (at calculation flows).

Regarding the mode of the network flows throughout the year, the frequency chart of the requested flows is allowed, presumably the same for all consumers. In the case shown in fig. 1, for instance: during $35 \%$ of the year (i.e. $035 \cdot 8760=3066$ ) it is requested between 50 and $75 \%$ of the design flow q, having an average value per class of $q k=0,625 \cdot q$. Let $K$ be the number of flow classes with the average values per class $\mathrm{qk}$ (in $\mathrm{m}^{3} / \mathrm{h}$ ) and the requesting time $\Delta \mathrm{tk}$ (in $\mathrm{h} /$ year ), for $\mathrm{k}=1,2, \ldots, \mathrm{K}$

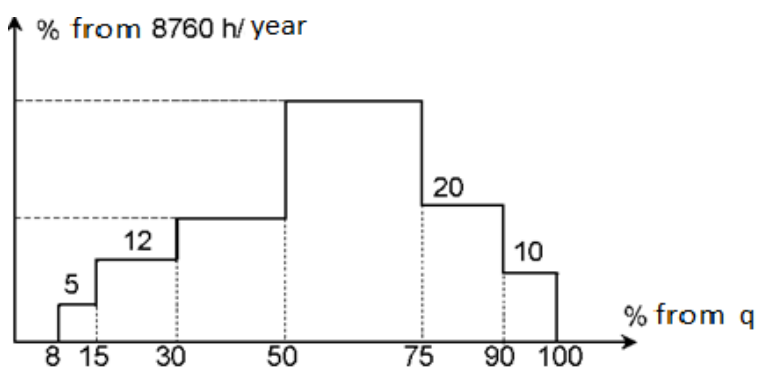

Figure 1 Example of frequency graph (as a fraction of the annual duration) of consumption flows (as fractions of the calculation flow q) [3]

In the case of each possible design solution, $\varphi$, after calculating the contribution of the pipes to the annual 
Journal of Marine Technology and Environment costs using the relation (1), $\mathrm{K}$ runs of the network hydraulic mode are performed, on the diameters of the solution $\varphi$, but successively taking into account the consumption flows from the nodes with values $\mathrm{qk}, \mathrm{k}=$ $1, \mathrm{~K}$. For each run there is a corresponding flow from the tank, QRk (in $\mathrm{m} 3 / \mathrm{h}$ ) and, through an iterative process, the concentration of chlorine entering the network is determined, CRk (in $\mathrm{mg} / \mathrm{l}$ ), which makes it possible to reach the minimum allowed value, Clim (în $\mathrm{mg} / \mathrm{l}$ ), of the residual chlorine, in the most disadvantaged node of the system. Next, the annual cost for chlorine treatment in the tank for solution $\varphi, V_{c}(\varphi)$, is obtained using the relation:

$$
{ }_{C}(\varphi)=10^{-3} \mathrm{v}_{\mathrm{C}} \frac{1}{\mathrm{~T}} \cdot \sum_{\mathrm{k}=1}^{\mathrm{K}} \mathrm{Q}_{\mathrm{Rk}} \cdot \Delta \mathrm{t}_{\Delta \mathrm{k}} \cdot \mathrm{C}_{\mathrm{R}_{\mathrm{k}}}
$$

where $\mathrm{V}_{\mathrm{c}}$ is the cost implied by producing / handling / injecting one kilogram of chlorine that is able to ensure the necessary concentrations $\mathrm{C}_{\mathrm{Rk}}$ when leaving the tank. The total annual costs for the construction of pipes and for the chlorination of water at the source, in the case of a possible design solution, $\varphi$, is obtained by adding (1) and (2), meaning:

$$
V_{h}(\varphi)=V_{h}(\varphi)+V_{C}(\varphi)
$$

and the performance function targeted in the optimal sizing model is:

$$
\min \left\{\left(V_{h}(\varphi)\right\}\right.
$$

Obviously, for each combination of standard diameters of the various pipes in the network, there are corresponding pipe costs, minimum loads in the network, chlorine concentrations at the source, on the required flow classes and, respectively, different chlorination costs to ensure the compliance with the requirements for water quality.

\section{Year 2021, Vol.I}

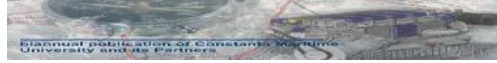

In order to view the subject matter in the AMF logic framework, to solve it and to provide a qualitative interpretation of the results, the authors based their work on the distribution network of Hanoi, used in the literature, as described in the chart below [2]. The lengths of the 34 pipes in the network and the serial numbers is the usable diameters are shown in table 2 , while table 3 contains the design flows $q$ (in $\mathrm{m} 3 / \mathrm{h}$ ) stressed in the network nodes. The network is allowed flat, with the load at the tank $\mathrm{Hr}=100 \mathrm{~m}$ and the minimum allowable load in nodes of $\mathrm{Hmin}=30 \mathrm{~m}$.

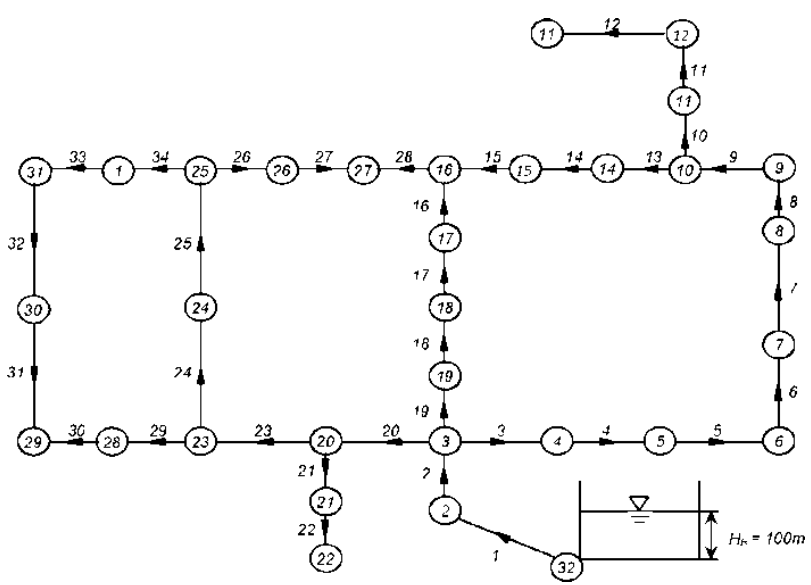

Figure 2 Diagram of the pipe distribution network in Hanoi (the directions on the pipes correspond to the situation marked as * in table 2)

Table 1. Usable standard diameters and corresponding unit costs

\begin{tabular}{|l|l|l|l|l|l|l|l|l|}
\hline Number & 1 & 2 & 3 & 4 & 5 & 6 & 7 & 8 \\
\hline$d_{s}(\mathrm{~mm})$ & 254 & 304.8 & 406.4 & 508 & 609.6 & 762 & 1016 & 1270 \\
\hline$c_{s}(\$ / \mathrm{m})$ & 33.39 & 45.726 & 70.4 & 98.378 & 129.333 & 180.748 & 278.78 & 450.9 \\
\hline
\end{tabular}

Table 2. Pipe lengths and allotted standard diameters [3]

\begin{tabular}{|c|c|c|c|c|c|c|c|c|c|c|c|}
\hline Pipe & $\begin{array}{c}\text { Length } \\
(m)\end{array}$ & \multicolumn{3}{|c|}{$d_{s}$ numbers in the list } & Pipe & \multicolumn{2}{c|}{$\begin{array}{c}\text { Length } \\
(m)\end{array}$} & \multicolumn{3}{|c|}{$d_{s}$ numbers in the list } \\
\hline 1 & 100 & 5 & 6 & $(7)$ & $8^{*}$ & 18 & 800 & 2 & 3 & 4 & $(5)$ \\
\hline 7 & 850 & 5 & $(6)^{*}$ & 7 & 8 & 24 & 1230 & 3 & 4 & 5 & $(6)$ \\
\hline 8 & 850 & 5 & $(6)^{*}$ & 7 & 8 & 25 & 1300 & 4 & $5^{*}$ & $(6)$ & 7 \\
\hline 9 & 800 & 5 & $(6)^{*}$ & 7 & 8 & 26 & 850 & 2 & $3^{*}$ & $(4)$ & 5 \\
\hline 10 & 950 & $5^{*}$ & 6 & $(7)$ & 8 & 27 & 300 & $(1)^{*}$ & 2 & 3 & 4 \\
\hline 11 & 1200 & 3 & 4 & $(5)^{*}$ & 6 & 28 & 750 & $(1)^{*}$ & 2 & 3 & 4 \\
\hline 12 & 3500 & 3 & $(4)^{*}$ & 5 & 6 & 29 & 1500 & 1 & 3 & $(3)^{*}$ & 4 \\
\hline 13 & 800 & $3 *$ & 4 & $(5)$ & 6 & 30 & 2000 & $(1)$ & $2^{*}$ & 3 & 4 \\
\hline 14 & 500 & 1 & $(2)$ & $3^{*}$ & 4 & 31 & 1600 & $(1)$ & $2^{*}$ & 3 & 4 \\
\hline 15 & 550 & $(1)$ & 2 & 3 & $4^{*}$ & 32 & 150 & 1 & $2^{*}$ & $(3)$ & 4 \\
\hline 16 & 2730 & $1 *$ & $(2)$ & 3 & 4 & 33 & 860 & 1 & $2^{*}$ & $(3)$ & 4 \\
\hline 17 & 2750 & 1 & 2 & $(3)^{*}$ & 4 & 34 & 950 & 3 & $(4)$ & $5^{*}$ & 6 \\
\hline
\end{tabular}


* - values selected in a suboptimal solution with

() - values selected in a suboptimal solution with AG (Popa and Tudor, 2000) [3]

Table 3. Design flows in junctions [3]

\begin{tabular}{|c|c|c|c|c|c|c|c|}
\hline $\begin{array}{c}\text { Nod } \\
\mathrm{e}\end{array}$ & $\left(\mathrm{m}^{3} / \mathrm{h}\right)$ & $\begin{array}{c}\text { Nod } \\
\mathrm{e}\end{array}$ & $\left(\mathrm{m}^{3}\right)$ & $\begin{array}{c}\text { Nod } \\
\mathrm{e}\end{array}$ & $\left(\mathrm{m}^{3} / \mathrm{h}\right)$ & $\begin{array}{c}\text { Nod } \\
\mathrm{e}\end{array}$ & $\left(\mathrm{m}^{3} / \mathrm{h}\right)$ \\
\hline 1 & 805 & 9 & 525 & 17 & 865 & 25 & 170 \\
\hline 2 & 890 & 10 & 525 & 18 & 1345 & 26 & 900 \\
\hline 3 & 850 & 11 & 500 & 19 & 60 & 27 & 370 \\
\hline 4 & 130 & 12 & 560 & 20 & 1275 & 28 & 290 \\
\hline 5 & 725 & 13 & 940 & 21 & 930 & 29 & 360 \\
\hline 6 & 1005 & 14 & 615 & 22 & 485 & 30 & 360 \\
\hline 7 & 1350 & 15 & 280 & 23 & 1045 & 31 & 105 \\
\hline 8 & 550 & 16 & 310 & 24 & 820 & 32 & $1 \overline{9}$ \\
\hline
\end{tabular}

Moreover, it was allowed a frequency chart of the flows stressed from consumers with $\mathrm{K}=5$ classes and the average values per class, respectively the stress durations throughout the year, as shown in table 4.
Table 4. Average consumer flows and demand times, by frequency classes [3]

\begin{tabular}{|c|c|c|c|c|c|}
\hline Class & 1 & 2 & 3 & 4 & 5 \\
\hline Fraction of q & 0.15 & 0.3 & 0.5 & 0.75 & 0.95 \\
\hline Duration (h) & 1463 & 1095 & 4380 & 1095 & 727 \\
\hline
\end{tabular}

\section{INTERPRETATION OF RESULTS}

The best solution among those found with these input data corresponds to total annual costs amounting to 278547,4 out of which $181439,7 /$ year is the cost of the pipes and 97107,7/year is the cost of water treatment at the tank. With a lifespan of $\mathrm{T}=30$ years, the total cost of the pipelines amounts to 5443191 - so it is lower than the best solution obtained with AG. The pipe diameters corresponding to this solution are marked with $*$ in table 2 and - for the sake of a comparison, the diameters of the optimal solution identified using AG in Popa and Tudor, 2000 are marked with parentheses (). It can be seen that, out of the approximately $39,5 \mathrm{~km}$ of pipeline network, $24.23 \mathrm{~km}$ had identical diameters as both the $\mathrm{AG}$ and the AMF formulations [3].

Table 5 shows the pipe flows, loads and chlorine concentrations in nodes for the solution marked with * in the design flow mode.

Table 5. Pipe flows, load and chlorine concentration in nodes in the design flow mode

\begin{tabular}{|l|l|l|l|l|l|l|l|l|l|}
\hline Pipe & $\begin{array}{l}\text { Debit } \\
(\mathrm{m} 3 / \mathrm{h})\end{array}$ & Pipe & $\begin{array}{l}\text { Debit } \\
(\mathrm{m} 3 / \mathrm{h})\end{array}$ & Node & $\begin{array}{l}\text { Pressure } \\
(\mathrm{m})\end{array}$ & $\begin{array}{l}\text { Conc. } \\
(\mathrm{mg} / \mathrm{l})\end{array}$ & Node & $\begin{array}{l}\text { Pressure } \\
(\mathrm{m})\end{array}$ & $\begin{array}{l}\text { Conc. } \\
(\mathrm{mg} / \mathrm{l})\end{array}$ \\
\hline 1 & 19940.2 & 18 & 2453.4 & 1 & 38.24 & 0.271 & 17 & 52.98 & 0.290 \\
\hline 2 & 19050.2 & 19 & 2513.4 & 2 & 99.00 & 0.303 & 18 & 73.54 & 0.296 \\
\hline 3 & 8000.3 & 20 & 7686.4 & 3 & 86.63 & 0.300 & 19 & 79.09 & 0.299 \\
\hline 4 & 7870.3 & 21 & 1415.0 & 4 & 81.99 & 0.297 & 20 & 76.16 & 0.293 \\
\hline 5 & 7145.3 & 22 & 485.0 & 5 & 76.25 & 0.294 & 21 & 47.44 & 0.289 \\
\hline 6 & 6140.3 & 23 & 4996.4 & 6 & 70.29 & 0.289 & 22 & 34.30 & 0.288 \\
\hline 7 & 4790.3 & 24 & 3245.4 & 7 & 64.18 & 0.288 & 23 & 53.71 & 0.286 \\
\hline 8 & 4240.3 & 25 & 2425.4 & 8 & 57.15 & 0.286 & 24 & 48.81 & 0.281 \\
\hline 9 & 3715.3 & 26 & 1041.3 & 9 & 51.65 & 0.283 & 25 & 40.00 & 0.277 \\
\hline 10 & 2000.0 & 27 & 141.3 & 10 & 47.67 & 0.280 & 26 & 31.19 & 0.274 \\
\hline 11 & 1500.0 & 28 & 228.7 & 11 & 43.29 & 0.276 & 27 & 30.52 & 0.267 \\
\hline 12 & 940.0 & 29 & 706.1 & 12 & 40.18 & 0.270 & 28 & 46.55 & 0.278 \\
\hline 13 & 1190.3 & 30 & 416.1 & 13 & 30.96 & 0.250 & 29 & 31.65 & 0.269 \\
\hline 14 & 575.3 & 31 & 56.1 & 14 & 36.83 & 0.278 & 30 & 31.43 & 0.258 \\
\hline 15 & 295.3 & 32 & 303.9 & 15 & 35.07 & 0.275 & 31 & 32.05 & 0.266 \\
\hline 16 & 243.4 & 33 & 408.9 & 16 & 34.90 & 0.269 & 32 & 100.00 & 0.303 \\
\hline 17 & 1108.4 & 34 & 1214.1 & - & - & - & - & - & - \\
\hline
\end{tabular}

It may be seen that the minimum value imposed for the residual chlorine in the network, $C_{\lim }=0,25 \mathrm{mg} / \mathrm{l}$, is reached in node 13 , while the minimum pressure load value of $30,52 \mathrm{~m}$ from node 27 is higher than the limit imposed by $\mathrm{H}_{\min }=30 \mathrm{~m}$. The chlorine concentration necessary when leaving the tank, in the design flow mode, is $C_{R}=0,303 \mathrm{mg} / 1$.
When the network is running, with the average consumption flows by classes as shown in table 4 , it turned out that the critical node in terms of water quality remains node 13 , and in order to ensure in this node 
$\mathrm{C}_{\lim }=0,25 \mathrm{mg} / \mathrm{l}$, the chlorine concentrations

necessary when leaving the tank are shown in table 6.

Table 6. Chlorine concentration when leaving the tank, by flow classes

\begin{tabular}{|c|c|c|c|c|c|}
\hline Class & 1 & 2 & 3 & 4 & 5 \\
\hline $\begin{array}{c}\text { Average flow in the } \\
\text { network (m3/h }\end{array}$ & 2991 & 5982 & 9970 & 14955 & 18943 \\
\hline $\begin{array}{c}\text { Chlorine } \\
\text { concentration in } \\
\text { tank (mg / })\end{array}$ & 0.863 & 0.470 & 0.367 & 0.323 & 0.306 \\
\hline
\end{tabular}

It can be seen that, for the considered network and input data, only at the lowest consumption flows (15\% of the calculation values) the chlorine concentration necessary when leaving the tank exceeds the upper limit of $0,5 \mathrm{mg} / \mathrm{l}$ stated by law no. 458/2002 [5]. For this specific situation it would be useful to address the issue of finding the optimum positions and chlorine concentrations introduced in the network by additional booster stations, so as it does not exceed the maximum accepted value when leaving the tank and so as it does not fall below the minimum accepted value when it is already in the network [3].

\section{CONCLUSION}

Approaches based on Artificial Intelligence are a strong and very effective tool when modelling water distribution projects. When used, the model can successfully simulate tank emptying, transit flows, node pressures and future conditions. However, this work method based on models identifies the various stresses in order to analyze them and, ultimately, to find the best solutions. This example shown by the two authors can be adapted and calibrated to particular situations, which substantiates decision making, but can also be a reference for addressing the problem using other artificial intelligence techniques.

\section{REFERENCES}

[1] Ianculescu O., Ionescu G., Alimentari cu apa, Editura Matrix Rom, Bucharest, 2002, 127 - 142

[2] M van Dijk, SJ van Vuuren, JE van Zyl, Optimising water distribution systems using a weighted penalty in a genetic algorithm, Water SA Vol. 34 No. 5 October 2008, 537 - 548

[3] Vuţă L. I., Popa R., Model de dimensionare optimă a reţelelor hidraulice, ţinând seama de calitatea apei, IWM Conference (2008), 135 - 145

[4]

https://ro.qaz.wiki/wiki/Ant_colony_optimization_algori thms accessed at 25.02.2021

[5] LEGE nr. 458 din 8 iulie 2002 (**republicată**) 